\title{
Broadband excitation in solid-state NMR of paramagnetic samples using Delays Alternating with Nutation for Tailored Excitation ('Para-DANTE')
}

\author{
Diego Carnevale ${ }^{\mathrm{a}, *}$, Veronika Vitzthum $^{\mathrm{a}}$, Olivier Lafon ${ }^{\mathrm{b}}$, Julien Trébosc ${ }^{\mathrm{b}}$, Jean-Paul Amoureux ${ }^{\mathrm{b}}$, \\ Geoffrey Bodenhausen ${ }^{\text {a,c,d,e }}$
}

\author{
a Institute des Sciences et Ingénierie Chimiques (ISIC), Ecole Polytechnique Fédérale de Lausanne (EPFL), 1015 Lausanne, Switzerland \\ ${ }^{\mathrm{b}}$ Unité de Catalyse et de Chimie du Solide (UCCS), UMR 8181 CNRS, Université de Lille, 59652 Villeneuve d'Ascq, France \\ ${ }^{\mathrm{C}}$ Département de Chimie, Ecole Normale Supérieure, 75231 Paris Cedex 05, France \\ d UMR 7203, CNRS/UPMC/ENS, Paris, France \\ e Université de Pierre-et-Marie Curie, Paris, France
}

\section{A R T I C L E I N F O}

\section{Article history:}

Received 17 May 2012

In final form 26 September 2012

Available online 2 October 2012

\begin{abstract}
A B S T R A C T
This Letter shows that interleaved sequences of short pulses in the manner of 'Delays Alternating with Nutation for Tailored Excitation' (DANTE) with $N=1,2,3 \ldots$ equidistant pulses per rotor period extending over $K$ rotor periods can be used to excite, invert or refocus a large number of spinning sidebands of spin$1 / 2$ nuclei in paramagnetic samples where hyperfine couplings lead to very broad spectra that extend over more than $1 \mathrm{MHz}$. The breadth of the response is maintained for $r f$-field amplitudes as low as $30 \mathrm{kHz}$ since it results from cumulative effects of individual pulses with very short durations.
\end{abstract}

(c) 2012 Elsevier B.V. All rights reserved.

\section{Introduction}

Magic-angle spinning (MAS) is widely used [1-3] in nuclear magnetic resonance (NMR) to average first-order anisotropic interactions such as chemical shift anisotropies (CSA's), quadrupole interactions, and homo- or hetero-nuclear dipole-dipole interactions. Indeed, when the spinning rate is much faster that the magnitude of the anisotropy of these interactions, only their isotropic parts remain [4]. On the other hand, if the spinning rate is not too fast, spinning sidebands remain, and the principal components of the interaction tensors can be determined from the intensities of these sidebands [5-6]. This information can then be compared with DFT calculations of NMR parameters to obtain unambiguous spectral assignments and to investigate disorder in position or composition [7-13].

Today's state-of-the-art MAS probes allow for spinning rates up to $v_{\text {rot }}=110 \mathrm{kHz}$. For most second-rank orientation-dependent interactions, such as ${ }^{13} \mathrm{C}-{ }^{1} \mathrm{H}$ dipolar couplings and chemical shielding tensors, such spinning rates are sufficient to observe spectra that are dramatically simplified like in isotropic phase. Other anisotropic interactions, such as paramagnetic shifts [14,15] and quadrupolar couplings [16], may be far larger than the highest spinning rates that can be achieved, leading to extensive families of spinning sidebands.

In paramagnetic solids, the excitation of transverse magnetization can be achieved in the usual manner by applying a short

\footnotetext{
* Corresponding author. Fax: +41216939435.

E-mail address: diego.carnevale@epfl.ch (D. Carnevale).
}

rectangular pulse immediately followed by the acquisition of the free induction decay. However, significant frequency-dependent phase errors may build up during the dead time prior to acquisition [17], and a first-order phase correction may lead to severe baseline distortions. In principle, frequency-dependent phase errors can be refocused efficiently by spin-echoes [18,19]. Indeed, the phase acquired by a coherence of order $p=+1$ in a defocusing period $\tau$ can be cancelled in a refocusing period $\tau^{\prime}$ if the coherence order $p=-1$ is selected in this interval by a suitable phase cycle. However, applying refocusing pulses to very wide spectra was hitherto regarded as technically very challenging, since it normally requires very intense radio-frequency $(r f)$ pulses.

In order to compare the efficiency of various excitation schemes for a given anisotropic interaction $A$, we introduce the dimensionless ratio, $\Lambda_{A}=v_{1} / \Delta_{A}$, where $v_{1}$ is the $r f$-field strength in $\mathrm{Hz}$ and $\Delta_{A}=A_{z z}-A_{i s o}$ expresses the anisotropy, also in units of $\mathrm{Hz}, A_{z z}$ and $A_{i s o}$ being the largest principal value and the isotropic component, respectively, of the tensor $A$. Excitation methods that are efficient for weak $r f$ fields and small $\Lambda_{A}$ values are highly desirable, especially for low- $\gamma$ nuclei where high $r$-field strengths are difficult to achieve. Uniform broadband excitation is important (i) to determine accurate principal values of the interaction tensors, (ii) to increase the $S / N$ ratio, and (iii) for multi-dimensional experiments.

Excitation with short rotor-synchronized pulses using Delays Alternating with Nutation for Tailored Excitation (DANTE) [20-23], allows one to overcome the limitations due to weak $r f$ fields. DANTE excitation can provide a uniform broadband excitation of very wide spectra as encountered in paramagnetic samples, 
thus retaining the information about the principal components of the tensors encoded in the spinning sideband intensities.

In the most common case where the spin-lattice relaxation of the unpaired electrons is fast on the NMR timescale, one only needs to consider the thermally averaged electron moment. The resulting inhomogeneous broadening of nearby nuclear spins is then determined by the dipolar coupling between the nuclear moments and the thermally averaged electron moment [14]. The anisotropic part of this interaction can be described by an electron-nucleus dipolar coupling tensor, which is formally equivalent to the familiar tensor of chemical shielding anisotropy [24,25]. The well-known Herzfeld-Berger approach [6] can thus be used to determine the principal components of the electron-nucleus dipolar coupling tensor by analyzing the intensities of the spinning sidebands.

The excitation or inversion of very broad ranges of anisotropic chemical sites in solid-state NMR has been addressed by several authors. In static samples, perhaps the most successful approaches use (WURST) refocusing pulses [26] in combination with Car-Purcell Meiboom-Gill (CPMG) schemes for spins $I=1 / 2$, and with quadrupolar QCPMG schemes for spins $I>1 / 2$ [27-28]. In samples spinning at the magic angle as well as for static samples, adiabatic pulses such as SHAP [25] and $S^{3} A P$ [29] schemes have been proposed to achieve broadband inversion. The use of pulses shorter than $90^{\circ}$ and $180^{\circ}$ for uniform excitation of inhomogeneously broadened spectra have also been employed in stimulated echoes and MAT-PASS experiments [30].

In this Letter, we propose the use of DANTE schemes of rotorsynchronized pulses for both excitation and inversion of severely broadened spectra of paramagnetic samples. We show that the method is efficient at very low $r$ f-field strengths of about $30 \mathrm{kHz}$. We also prove that any cumulative flip angle can be achieved in such manner.

\section{Results and discussion}

In $\mathrm{CeF}_{3}$, two distinct ${ }^{19} \mathrm{~F}$ sites have been observed [31] in a MAS spectrum recorded at $9.4 \mathrm{~T}\left(376.3 \mathrm{MHz}\right.$ for $\left.{ }^{19} \mathrm{~F}\right)$ with a $2.5 \mathrm{~mm}$ rotor and a spinning frequency $v_{\text {rot }}=35 \mathrm{kHz}$, using a single short pulse of duration $\tau_{p}=0.5 \mu$ s [31]. The spectrum features two sets of interleaved and partially overlapping spinning sidebands that are separated by $33.6 \mathrm{ppm}=12.6 \mathrm{kHz}$, extending over more than $1000 \mathrm{ppm}$ or $380 \mathrm{kHz}$. We shall refer to these two ${ }^{19} \mathrm{~F}$ sites in $\mathrm{CeF}_{3}$ as $\mathrm{A}$ and $\mathrm{B}$, corresponding to low and high frequencies (i.e., from right to left in the spectra).

All ${ }^{19} \mathrm{~F}$ MAS spectra of $\mathrm{CeF}_{3}$ shown in this Letter have been recorded on a Bruker 800 spectrometer (18.8 T, 752.6 MHz for ${ }^{19} \mathrm{~F}$ ) with an Avance-III console, using a $1.3 \mathrm{~mm}$ double resonance MAS probe, and a spinning frequency $v_{\text {rot }}=60 \mathrm{kHz}$. At this field, the two species are separated by $33.6 \mathrm{ppm}=25.3 \mathrm{kHz}$. The fluorine chemical shifts were referenced to $\mathrm{CaF}_{2}$ at $-108.6 \mathrm{ppm}$ [32]. The spectra shown in Figure 1 have been recorded with an $r f$-field amplitude $v_{1}=227 \mathrm{kHz}$. The MAS spectrum recorded after a single rectangular pulse of duration $\tau_{p}=1.1 \mu$ s (nutation angle $\beta=90^{\circ}$ ) is shown in Figure $1 \mathrm{a}$. The ${ }^{19} \mathrm{~F}$ background signal from the Teflon cable of the probe at about $-130 \mathrm{ppm}$ interferes with the resonances of the $\mathrm{CeF}_{3}$ sample. Because of the dead-time of $5 \mu \mathrm{s}$, the spectrum of Figure 1a cannot be deconvoluted assuming two different isotropic chemical shifts and anisotropic paramagnetic interactions.

Using an echo allows one to cancel the broad signal from the probe and to avoid distortions due to the dead-time. Figure 1b shows a spectrum obtained with a $90^{\circ}-\tau-180^{\circ}-\tau^{\prime}$ spin-echo, using two rectangular pulses with durations $\tau_{p}=1.1$ and $2.2 \mu$ s. Hyperfine couplings to the paramagnetic $\mathrm{Ce}^{3+}$ ions result in a very wide
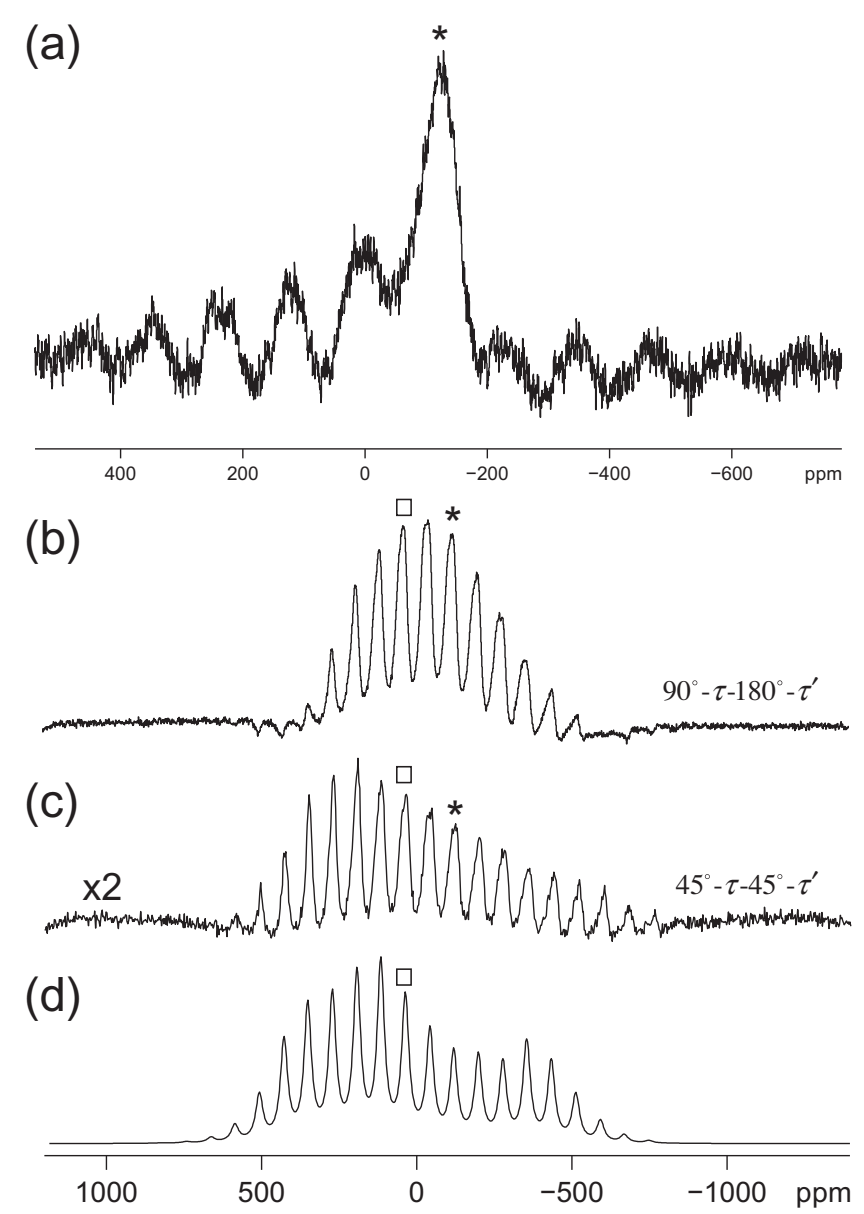

Figure 1. Experimental ${ }^{19} \mathrm{~F}$ MAS spectra of $\mathrm{CeF}_{3}$ recorded at $18.8 \mathrm{~T}$ with $v_{\text {rot }}=60$ $\mathrm{kHz}$, an $r$-field strength $v_{1}=227 \mathrm{kHz}$ and a recycling delay $t_{R D}=0.3 \mathrm{~s}$. (a) Spectrum obtained by Fourier transformation of a free induction decay acquired after a dead time of $5 \mu \mathrm{s}$. One single scan was acquired. (b) Spectrum resulting from the Fourier transformation of a spin-echo excited by a $90^{\circ}-\tau-180^{\circ}-\tau^{\prime}$ sequence with $\tau=\tau^{\prime}=\tau_{\text {rot }}=16.67 \mu \mathrm{s}$. Thirty-two scans were acquired. (c) Spectrum resulting from a spin-echo excited by a $45^{\circ}-\tau-45^{\circ}-\tau^{\prime}$ sequence, again with $\tau=\tau^{\prime}=\tau_{\text {rot. }}$. (d) Spectrum simulated for $\delta_{\text {iso }}=35 \mathrm{ppm}, \Delta_{\text {aniso }}=-638 \mathrm{ppm}$ and $\eta=0.60$. Five hundred and twelve scans were acquired. Asterisks and squares indicate the carrier frequency and the isotropic shift, respectively.

spectrum that covers more than $900 \mathrm{kHz}$. Under these experimental conditions, the two species A and B separated by $25.3 \mathrm{kHz}$ cannot be resolved and one observes one single comb of spinning sidebands each with a full width at half-maximum (FWHM) of ca. $23 \mathrm{kHz}$. However, $T_{2}^{\prime}$ measurements performed on the right and left shoulder of the most intense peak confirm the existence of two environments with different relaxation rates $T_{2}^{\prime}=0.52 \mathrm{~ms}$ (site $A$ on the right) and $1.2 \mathrm{~ms}$ (site $B$ on the left). The envelope of the spinning sideband pattern is roughly symmetrical with respect to the carrier frequency marked by an asterisk at $-130.2 \mathrm{ppm}$, reflecting the sinc-envelope determined by the longest of the two pulses. Indeed, the outer sidebands appear in negative absorption, corresponding to wiggles of the sinc function. As shown in Figure 1c, if we shorten both pulses lengths to obtain a $45^{\circ}-\tau-45^{\circ}-\tau^{\prime}$ spin-echo experiment, this allows one to excite a much broader spectral range, albeit with an attenuated intensity and a decreased $S / N$ ratio. The total spectrum now extends over at least $1 \mathrm{MHz}$ and, in contrast to Figure $1 \mathrm{~b}$, clearly reveals a CSA-type pattern. The spinning sideband patterns of the two species are similar [31], so that the global envelope resembles that of a single species. The spectrum in Figure 1c shows a nearly flat excitation profile, without any negative sidebands that are 
characteristic of sinc wiggles. However, we should remember that a strong $r f$ field $v_{1}=227 \mathrm{kHz}$ has been used for all spectra in Figure 1 . Such a large $r f$-field strength is only possible for favorable Larmor frequencies as for ${ }^{19} \mathrm{~F}$ and small rotor diameters. A simulation of the spinning sideband envelope is shown in Figure 1d. The fitted parameters are $\delta_{\text {iso }}=35 \mathrm{ppm}, \Delta_{\text {aniso }}=-638 \mathrm{ppm}$ and $\eta=0.60$. Hence, the $\Lambda$ value achievable with a $45^{\circ}-\tau-45^{\circ}-\tau^{\prime}$ spin echo is 0.47 . It turns out that the isotropic shift in all spectra of Figure 1 is $v_{\text {iso }} \approx 120 \mathrm{kHz}$ (marked by a square), so that the carrier frequency (marked by an asterisk, *) coincides with the second sideband to the right of the isotropic shift.

Figure 2 shows spin-echoes excited and refocused by DANTE sequences applied to $\mathrm{CeF}_{3}$. The magnetization was first excited by a burst of $N$ equidistant pulses per rotor period $\tau_{\text {rot }}$, each individual pulse having a duration $\tau_{p}$, extending over $K$ rotor periods, with a cumulative flip angle near $\pi / 2$, and then refocused by a second burst with $N$ equidistant pulses per $\tau_{\text {rot }}$, again of duration $\tau_{p}$, but extending over $2 K$ rotor periods with a cumulative flip angle in the vicinity of $\pi$. We shall refer to such spin-echo sequences as $D_{N}^{K}-\tau-D_{N}^{2 K}-\tau^{\prime}$.

The DANTE spin-echo spectra shown in Figure 2 have been recorded with an $r f$-field amplitude $v_{1}=227 \mathrm{kHz}$. The carrier frequency $v_{r f}$ (indicated by an asterisk, $*$ ) has again been set to coincide with the second spinning sideband to the right of the isotropic shift (marked by a square) of the species $B$, which has the longest $T_{2}^{\prime}(\mathrm{B})=1.2 \mathrm{~ms}$. We have shown $[22,23]$ that the excitation profile of a single $D_{N}^{K}$ block (and by extension of a $D_{N}^{K}-\tau-D_{N}^{2 K}-\tau^{\prime}$ spin-echo sequence) comprises a manifold of $r f$ spikelets that occur at regular frequency intervals $N v_{\text {rot }}$ with respect to the carrier frequency $v_{r f}$. Figure 2 shows that only spinning sidebands spaced by $N v_{\text {rot }}$ are observed, as if the apparent spinning rate were $N v_{\text {rot }}$. However, the relative intensities of the spinning sidebands always reproduce those determined by the true spinning rate at which the experiments were performed. Thus, a $D_{2}^{K}$ sequence applied to a sample spinning at a rate $v_{\text {rot }}$ does not yield the same spinning sidebands as a $D_{1}^{2 K}$ sequence when spinning at $2 v_{\text {rot }}$. At a given spinning rate $v_{\text {rot }}$, a $D_{2}^{K}$ sequence simply produces half the spinning sidebands (with twice the spacing but the same relative intensities) as a $D_{1}^{2 K}$ sequence. It is also worth noting that, since the carrier frequency is positioned at $v_{r f}=v_{i s o}+2 v_{\text {rot }}$, there is no $r f$ spikelet that coincides with the isotropic shift $v_{\text {iso }}$ if $N>2$, and the spectra do not show any isotropic peak (Figure $2 \mathrm{c}$ and $\mathrm{d}$ ).

When comparing Figures 1 and 2, it is apparent that the linewidths of the sidebands are much narrower in the latter case. This is due to the selectivity of the spikelets generated by the spin-echo sequences. If we consider the scheme $D_{N}^{K}-\tau-D_{N}^{2 K}-\tau^{\prime}$ used to obtain the sideband patterns shown in Figure $2 a-d$, the total length of the two $r f$ pulses is $3 K \tau_{\text {rot }}$, and the line-widths of the peaks decrease with $K$ according to $F W H M \approx 1 /\left(3 K \tau_{\text {rot }}\right)$. These widths are much smaller than those observed in Figure 1, so that only a fraction of the width of the spinning sidebands is excited. We can thus conclude that in $\mathrm{CeF}_{3}$ the ${ }^{19} \mathrm{~F}$ nuclei experience an inhomogeneous distribution of environments. We have performed our measurements with the carrier frequency set to a sideband belonging to the most intense site $B$, which has the longest $T_{2}^{\prime}$. Given the width of the spikelets of the $r f$ excitation profiles produced by our DANTE echoes (the greatest width is $5 \mathrm{kHz}$ for $D_{4}^{4}-\tau-D_{4}^{8}-\tau^{\prime}$ in Figure $2 \mathrm{~d}$ ), the second species A, which is shifted by $25.3 \mathrm{kHz}$ to the right, is simply not excited in Figure 2. These results highlight two apparently contradictory aspects of excitation schemes based on rotor-synchronized combs of pulses, i.e., (i) the broad global bandwidth of the excitation is typical of 'hard' pulses of short duration $\tau_{p}$ and (ii) the selectivity of the excitation by each spikelet is reminiscent of 'soft' pulses of duration $K \tau_{\text {rot. }}$. Figure 2e shows a fit of the spectrum of Figure $2 \mathrm{a}$, assuming that there is only one ${ }^{19} \mathrm{~F}$ species. The quality of the fit justifies this hypothesis. The relevant param-
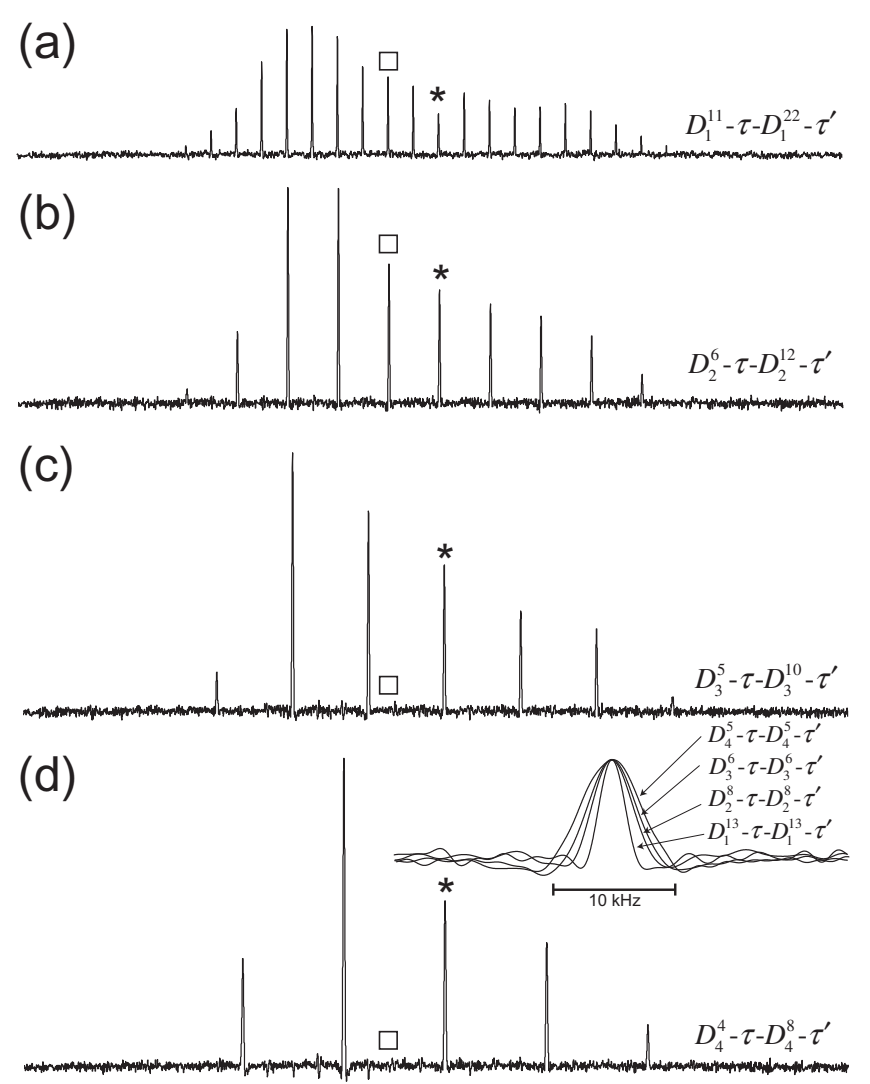

(e)

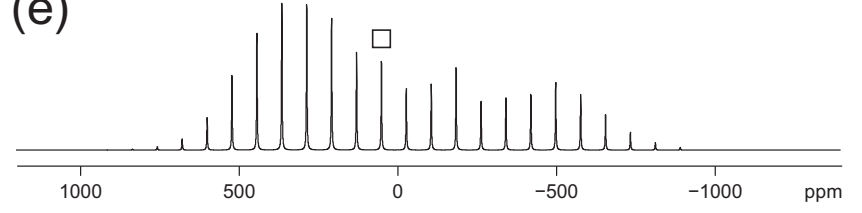

Figure 2. Experimental ${ }^{19} \mathrm{~F}$ MAS spectra of $\mathrm{CeF}_{3}$ obtained at $18.8 \mathrm{~T}$ with $v_{\text {rot }}=60$ $\mathrm{kHz}, v_{1}=227 \mathrm{kHz}$, and a recycling delay $t_{R D}=0.3 \mathrm{~s}$ by Fourier transformation of spin-echoes excited by $D_{N}^{K}-\tau-D_{N}^{2 K}-\tau^{\prime}$ sequences with $\tau=\tau^{\prime}=\tau_{\text {rot }}=16.67 \mu$ s comprising two bursts of DANTE pulses lasting $K$ and $2 K$ rotor periods, respectively, with $N=1,2,3$ and 4 pulses of duration $\tau_{p}=0.1 \mu$ s each per rotor period. The numbers of rotor periods $K=11,6,5$ and 4 were optimized empirically in (a-d). (e) Spectrum simulated for $\delta_{\text {iso }}=30 \mathrm{ppm}, \Delta_{\text {aniso }}=-814 \mathrm{ppm}, \eta=0.40$ and $\tau_{p}=0.1 \mu \mathrm{s}$. The inset shows peaks near the carrier frequency (with amplitudes scaled to the same vertical scale) obtained with spin-echoes excited by $D_{N}^{K}-\tau-D_{N}^{K}-\tau^{\prime}$ DANTE sequences with increasing $N=1,2,3$ and 4 and $K=5,6,8$, and 13 , with $v_{1}=227 \mathrm{kHz}$ and a constant duration $\tau_{p}=0.1 \mu$ s of the individual pulses. Asterisks indicate the carrier frequency and squares the isotropic shift. Thirty two scans were acquired in each case. All other experimental parameters were as in Figure 1.

eters obtained are $\delta_{\text {iso }}=30 \mathrm{ppm}, \Delta_{\text {aniso }}=-814 \mathrm{ppm}$ and $\eta=0.40$. This corresponds to $\Lambda=0.37$. The broadband DANTE excitation allows the determination of anisotropic components over a much wider range than single-pulse excitation. In the latter case, as shown in Figure 1c, one underestimates the anisotropy by ca. $200 \mathrm{ppm}$, since the apparent value is $\Delta_{\text {aniso }}=-638 \mathrm{ppm}$. Small differences between experimental and simulated spectra may be ascribed to the fact that only a fraction of the crystallites has been excited by DANTE spin-echoes. In Figure 2a, one observes 22 sidebands, and the full frequency range exceeds $1.26 \mathrm{MHz}$.

It is also possible to obtain echoes with two bursts of equal duration, i.e., using a $D_{N}^{K}-\tau-D_{N}^{K}-\tau^{\prime}$ sequence. In this manner, both bursts have the same duration $K \tau_{\text {rot }}$, and their spikelets have the same width. The inset in Figure 2d shows an overlay of the normalized peaks at the carrier frequency $v_{r f}$ obtained with $D_{N}^{K}-\tau-D_{N}^{K}-\tau^{\prime}$ for $N=1,2,3$ and 4 and $K=13,8,6$ and 5, respectively. Since the total length of the $r f$ pulses of the whole $D_{N}^{K}-\tau-D_{N}^{K}-\tau^{\prime}$ sequence decreases 
as $2 K \tau_{\text {rot }}=26 \tau_{\text {rot }}, 16 \tau_{\text {rot }}, 12 \tau_{\text {rot }}$ and $10 \tau_{\text {rot }}$, the full-width at halfmaximum (FWHM) of the peaks increases with $N$ according to $F W H M \approx 1 /\left(2 K \tau_{\text {rot }}\right)$. The line-widths $2.3,3.8,5.0$ and $6.0 \mathrm{kHz}$ of the peaks shown in the inset in Figure 2 agree with $K=13,8,6$ and 5 . The intensity of the on-resonance spinning sideband increases from a to $d$ because the number of rotor periods required for each pulse train decreases ( $K=11,6,5$ and 4 ). This was made possible by increasing $N$ from 1 to 4 . This leads to a reduction of the overall duration of the excitation and refocusing pulse sequences from $33,18,15$, to $12 \tau_{\text {rot }}$. As a result, the homogeneous decay is attenuated in going from a to $\mathrm{d}$, since it is proportional to $\exp \left\{-\left(3 K \tau_{\text {rot }}+2 \tau\right) / T_{2}^{\prime}\right\}$ in the sequence of Figure 2 .

Since spin-echo DANTE sequences are able to focus the $r f$ irradiation into narrow frequency spikelets, they allow one to excite many sidebands with a much lower $r f$-field amplitude than conventional spin-echo sequences using rectangular pulses. This advantage is proven experimentally in Figure 3, which compares spin-echo spectra recorded either with $45^{\circ}-\tau-45^{\circ}-\tau^{\prime}$ sequences (Figure 3a-e) or with $D_{1}^{K}-\tau-D_{1}^{K}-\tau^{\prime}$ sequences (Figure $3 \mathrm{f}-\mathrm{j}$ ) using $r f$ field amplitudes $v_{1}=227,114,57,29$ and $14 \mathrm{kHz}$. In each case the number $K$ of rotor periods was optimized experimentally. We have chosen $45^{\circ}-\tau-45^{\circ}-\tau^{\prime}$ rather than $90^{\circ}-\tau-180^{\circ}-\tau^{\prime}$ sequences because, as shown in Figure $1 \mathrm{~b}$ and $\mathrm{c}$ the latter sequence leads to large distortions, even with strong $r f$ fields. A progressive deterioration of the sideband envelope (Figure 3a-e), and hence of the information about the anisotropy, is observed with $45^{\circ}-\tau-45^{\circ}-\tau^{\prime}$ spin-echoes when the $r$-field amplitude is decreased. Moreover, as the $45^{\circ}$ pulse length increases with decreasing $r f$ field, the wings of the sideband envelope fall into the wiggling regions of the excitation profile, and negative sidebands become observable (Figure 3c-e). On the contrary, and remarkably, an almost complete preservation of the spinning sideband envelope is obtained
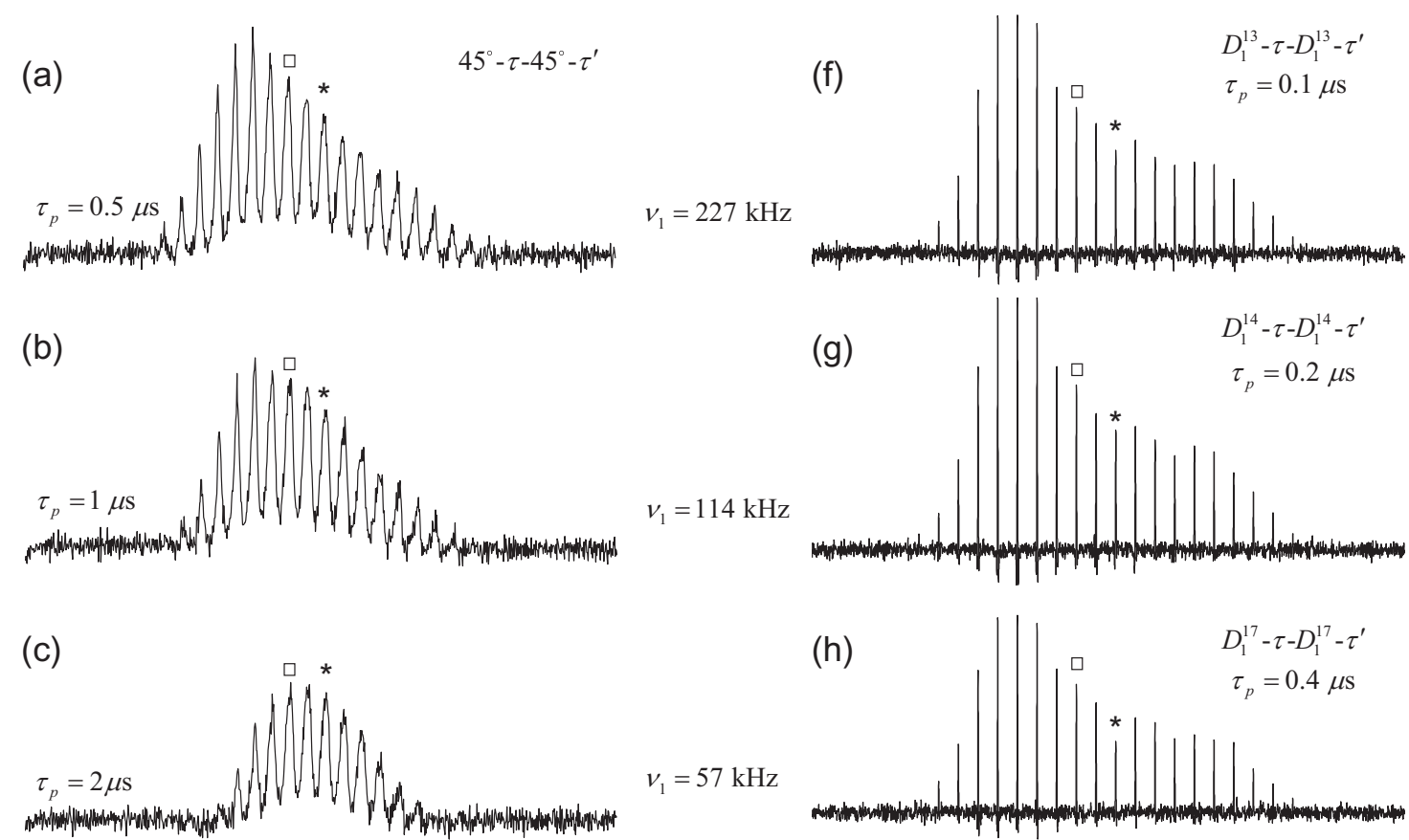

(d)

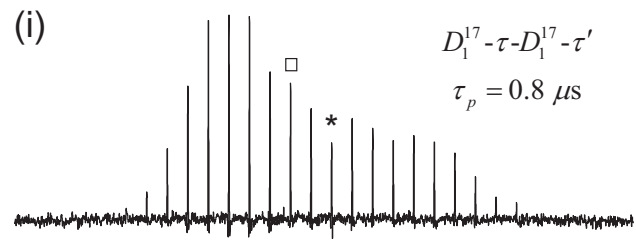

(e)

(j)

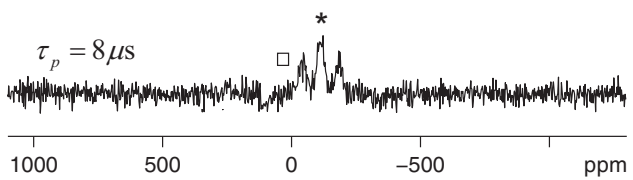

$v_{1}=14 \mathrm{kHz}$

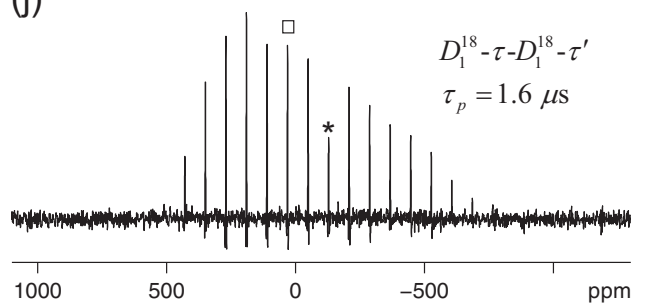

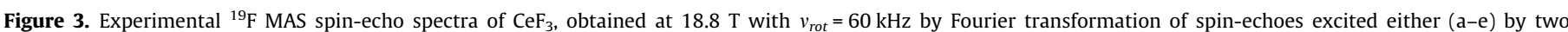

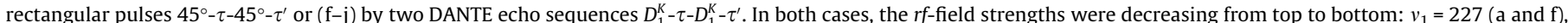

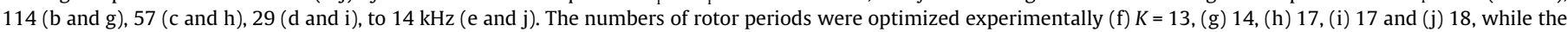

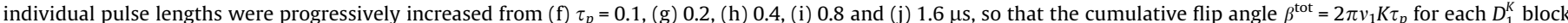

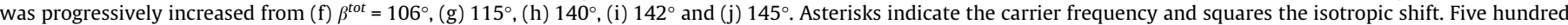
and twelve and 32 scans were acquired for spin echoes and DANTE echoes, respectively. All other experimental parameters were as in Figure 1. 
(a)

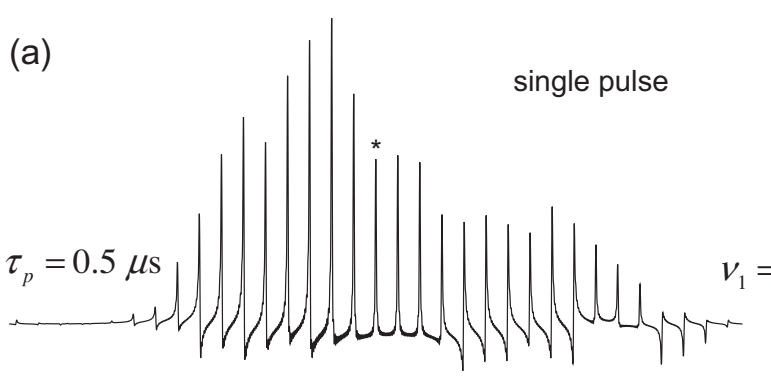

(b)

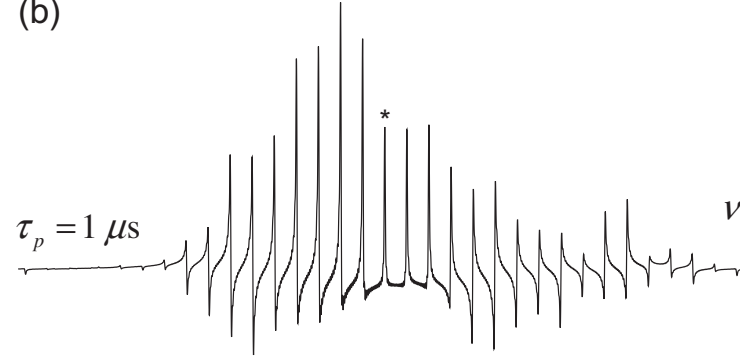

(c)

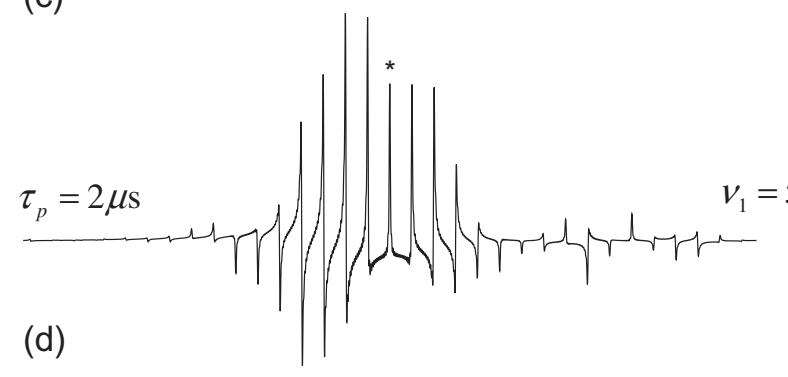

(d)

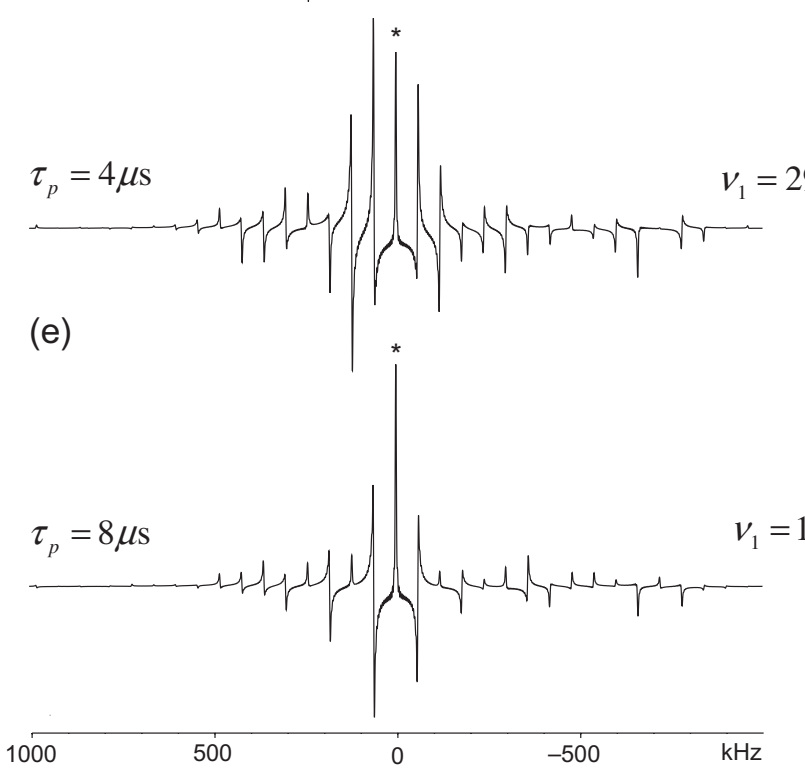

(f)

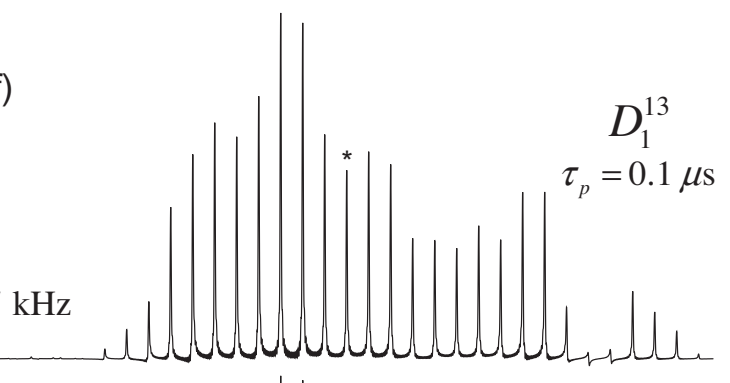

(g)

$v_{1}=114 \mathrm{kHz}$

(h)

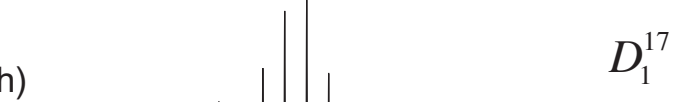

$D_{1}^{14}$

$\tau_{p}=0.2 \mu \mathrm{s}$ 
(a)

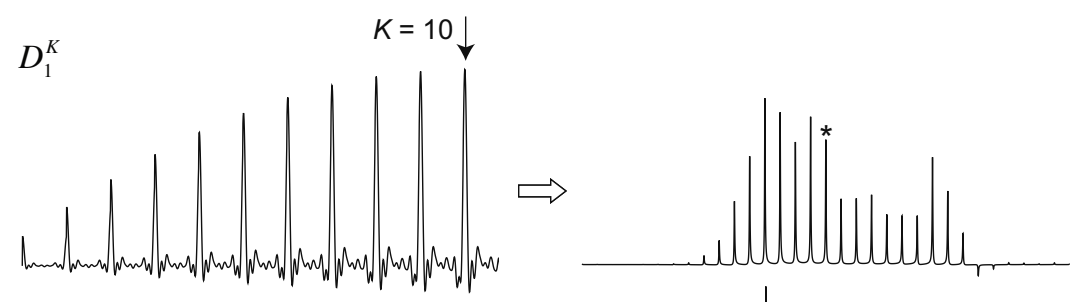

(b)
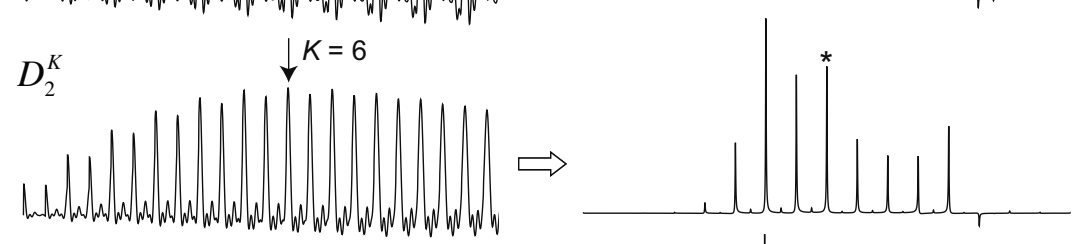

(c)

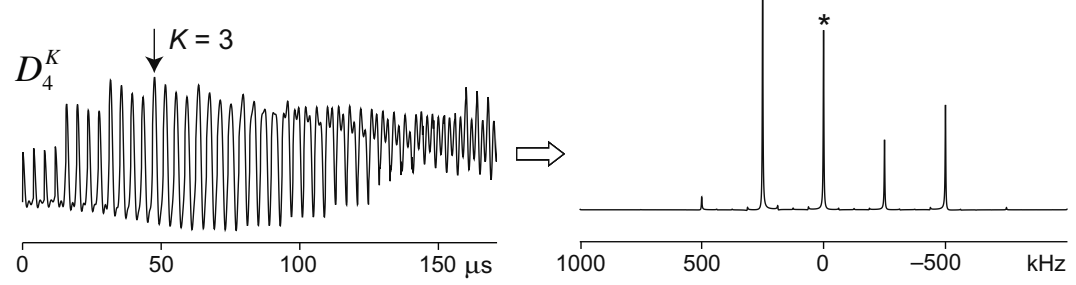

(d)

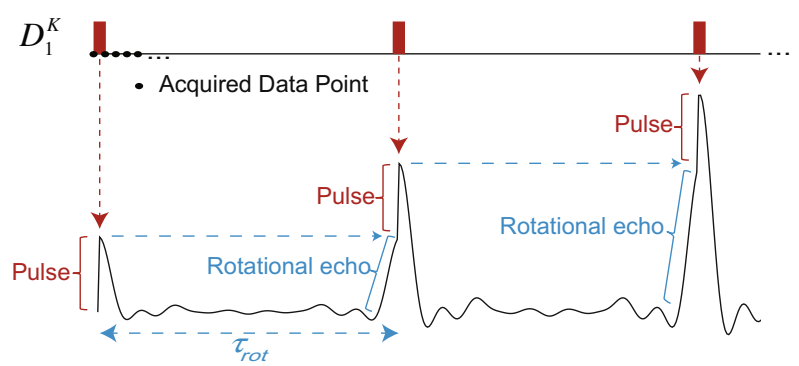

(e)

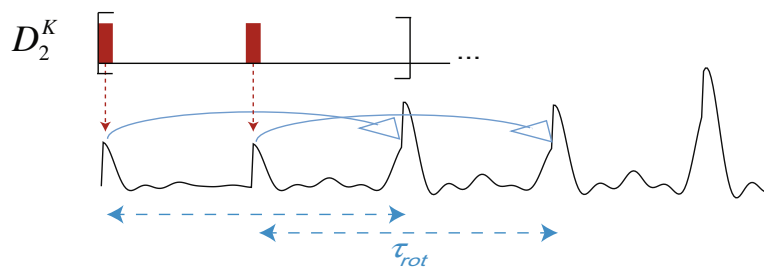

(f)

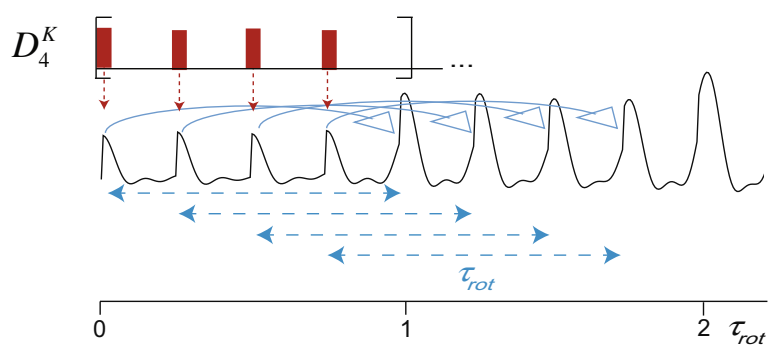

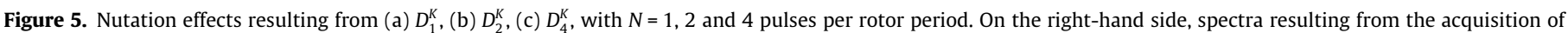

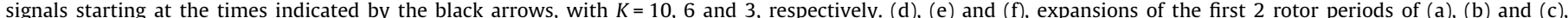

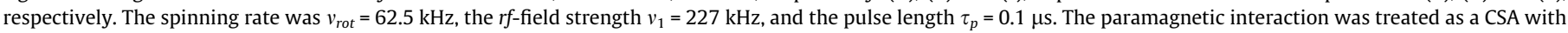

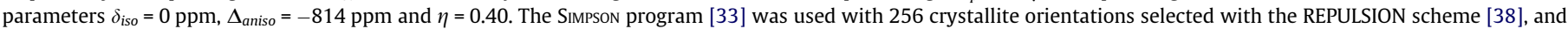
eight $\gamma$ angles were considered.

of Figure 2a $\left(\delta_{\text {iso }}=30 \mathrm{ppm}, \Delta_{\text {aniso }}=-814 \mathrm{ppm}\right.$ and $\left.\eta=0.40\right)$ are therefore reliable.

Numerical simulations have been carried out with SIMPSon [33] to corroborate the experimental findings shown in Figure 3. No dead time prior to acquisition is required in silico, so that refocusing can be dispensed with. Therefore, MAS spectra simulated for a single $D_{1}^{K}$ block and a single rectangular $45^{\circ}$ pulse can be compared directly in terms of breadth and uniformity of the excitation profile. As discussed above, the paramagnetic interaction can be treated like a CSA with $\Delta_{\text {aniso }}=-814 \mathrm{ppm}$ and $\eta=0.40$. The relevant parameters are the same as in Figure 3 . The resulting simulated MAS spectra are shown in Figure 4 . With a single $45^{\circ}$ pulse, the full spectrum can only be properly excited with the strongest $r f$ field (Figure 4a). Nevertheless, even this spectrum presents some distor- 
tions (compare Figure 4a and f), which worsen if one uses a spinecho with two $45^{\circ}$ pulses. On the contrary, the robustness of DANTE with respect to the $r f$-field strength is highlighted in the simulations of Figure $4 \mathrm{f}-\mathrm{j}$, which show a good agreement with the experimental DANTE spin-echo spectra of Figure 3. The progressive increase in phase distortion as the $r f$-field strength is lowered (and the pulse length $\tau_{p}$ increased) reflects the progressive increase in selectivity of the excitation schemes. With respect to such distortions, DANTE proves to be much more robust than single-pulse excitation. These simulations also prove that direct DANTE excitation does not allow significant frequency-dependent phase errors to build up by the time the acquisition begins. This is due to the synchronization of the rotational echoes induced by the mechanical rotation of the sample and the $r f$ burst of excitation. If a DANTE block is properly synchronized, so that each individual pulse is applied exactly when the rotor has the same phase, there will be no phase error, provided signal acquisition starts exactly at the beginning of the $(K+1)$-th rotor period. In our $\mathrm{CeF}_{3}$ sample, we have found however that a single DANTE sequence without refocusing does not remove the background signal from the Teflon cable.

The simulations of Figure 4 show the breadth of the excitation profile that one can achieve in MAS spectra with DANTE schemes. In order to get more insight into how DANTE sequences work during the excitation process, Figure $5 \mathrm{a}-\mathrm{c}$ shows simulations of the nutation effects resulting from sequences with $N=1,2$ and 4 pulses per rotor period (i.e., $D_{1}^{K}, D_{2}^{K}$ and $D_{4}^{K}$ ). The right-hand side shows spectra resulting from Fourier transformation of signals acquired after optimum numbers of $K=10,6$ and 3 rotor periods (indicated by black arrows). The asterisks indicate the carrier frequency, which in this Figure coincides with the isotropic shift. As previously observed, with increasing $N$, the best intensity is achieved with smaller values of $K$, i.e., $D_{1}^{10}, D_{2}^{6}$ and $D_{4}^{3}$. These values are very similar to those found experimentally in the DANTE spin echo experiments of Figures 2 and 3. Differences can be ascribed to relaxation processes that are not included in the simulations. Figure $5 \mathrm{~d}-\mathrm{f}$ shows enlargements of the signals observed in the first 2 rotor periods. Above these signals, red rectangles depict the DANTE pulses. In d, the first $r f$ pulse projects a fraction of the magnetization onto the transverse plane (red dashed arrow). The subsequent free evolution under the inhomogeneous interactions causes the dephasing of the single-quantum coherence. At the end of the rotor period, a rotational echo (indicated by blue arrows and accolades) is formed by refocusing the dephasing, thus reestablishing the same coherent state as at the end of the first pulse. At this point, the second pulse further causes the magnetization to nutate, thus augmenting the projection onto the transverse plane. The resulting coherent state is then subject again to defocussing and refocusing in the course of the mechanical rotation. By virtue of this synergy between $r f$ pulses and mechanical rotation, the expectation value of the observable transverse magnetization is progressively increased, like in conventional DANTE experiments, but with the characteristic breadth of excitation highlighted in Figures 3 and 4 . Figure $5 e-f$ shows the build-up for $N=2$ and 4 , which highlight a further aspect of DANTE. When more than one pulse is applied in each rotor period, each of them acts independently with respect to the previous one, so to project onto to the $x y$ plane an equal amount of magnetization. Each projection gives rise to its own rotational echo delayed by its own rotor period. The subsequent DANTE block allows each pulse to add more magnetization onto its own rotational train of echoes, thus resulting in families of $N$ independent DANTE effects. This effect seems to be compromised as $K$ becomes progressively larger in the case of $N=4$ shown in Figure 5c. These simulations also show that $N$ pulses acting in a rotor-synchronized fashion result in a modulation of the signal which is very similar to what can be achieved by MAS rotation at $N v_{\text {rot }}$.
The simulations shown in Figures 4 and 5 describe some of the virtues of DANTE excitation schemes. The wide breadth of the excitation is made possible by the use of very short pulses $\tau_{p}$ on the order of a few tenths of microseconds. The use of a single short pulse in conventional pulse-acquire or spin-echo experiments would result in very small flip angles and hence in weak signals. The DANTE scheme overcomes this limitation. The synergy between the pulse sequence and the mechanical rotation allows the build-up of coherences which results in cumulative flip angles that are $N K$ times larger than could be achieved by a single short pulse of duration $\tau_{p}$. However, the width of each spikelet in the excitation spectrum is proportional to $\left(K \tau_{r o t}\right)^{-1}$. Therefore, if homogeneous or inhomogeneous interactions broaden the signals to an extent that exceeds the width of the spikelet, only a portion of the linewidth will be excited. Any isotropic shift or distribution of shifts that fall outside of the spikelet will remain silent in the resulting spectrum. In principle, this can be alleviated by varying the amplitudes of the pulses that make up the pulse train, as will be discussed in a forthcoming paper.

The results presented here indicate that spin-echoes obtained by DANTE excitation and refocusing schemes can provide undistorted MAS spectra with much wider spectral widths than conventional rectangular pulses. Furthermore, this can be achieved with limited $r f$-field strengths. In the case of $\mathrm{CeF}_{3}$ at $18.8 \mathrm{~T}$, it was sufficient to use an $r f$ field $v_{1}=30 \mathrm{kHz}(\Lambda=0.05)$, whereas rectangular pulses required at least $v_{1}=227 \mathrm{kHz}$ (Figure $1 \mathrm{c}, \Lambda=0.47$ ). It is worth bearing in mind that the spectrum obtained with a conventional spin-echo at the highest $r f$-field strength available (Figure 1c) resulted in underestimating the anisotropy $\Delta_{\text {aniso }}$ by ca. 200 ppm.

The use of DANTE for broadband inversion is particularly interesting. Figure 6 shows the nutation profile obtained for $\mathrm{CeF}_{3}$ with a spin-echo sequence $D_{1}^{11}-\tau-D_{1}^{22}-\tau^{\prime}$ using an $r f$-field amplitude $v_{1}=227 \mathrm{kHz}$, by progressively increasing the pulse length $\tau_{p}$ in the first $D_{1}^{11}$ block, while $\tau_{p} \approx 0.11 \mu \mathrm{s}$ was kept invariant in the refocusing $D_{1}^{22}$ block. When $\tau_{p} \approx 0.11,0.33$ and $0.55 \mu \mathrm{s}$ in the first DANTE block (arrows in Figure 5), the responses shown below correspond to a $\beta-\tau-180^{\circ}-\tau^{\prime}$ spin-echo, with $\beta=90^{\circ}, 270^{\circ}$ and $450^{\circ}$. The anisotropic information in these spectra is globally conserved. The condition $\beta=180^{\circ}$ leads to a vanishing signal, as clearly identifiable for $\tau_{p} \approx 0.22 \mu \mathrm{s}$. Figure 6 proves that DANTE schemes can be used for any desired nutation angle, which will be useful for many advanced NMR methods such as satellite-transition magicangle spinning (ST-MAS) [34,35].

The optimal parameters of DANTE experiments are determined by the empirical time constant $T_{2}^{\prime}$ of the homogeneous decay of the system under investigation. If the inhomogeneous broadening is very large, very short pulses $\tau_{p}$ are recommended so as to fully excite the whole anisotropic pattern. High sensitivity can then be achieved with an appropriate number of $N$ pulses per rotor period extending over $K$ rotor periods to ensure the desired cumulative flip angle, which is proportional to $N K \tau_{p}$. If homogeneous losses are severe, i.e., if $T_{2}^{\prime}$ is short, one can choose a large $N$ and small $K$, to limit the overall duration of the pulse trains. One can also increase the pulse length $\tau_{p}$ to decrease $K$, albeit at the expense of the bandwidth. In any case, faster spinning rates decrease the effects of homogeneous decay.

The gain in sensitivity that one can obtain with DANTE sequences can be appreciated from Figure 3. The conventional spin echoes from a to e required averaging of 512 transients, whereas the DANTE echoes from $f$ to $j$ resulted from only 32 scans. Since the intensities of the spectra are comparable, the improvement in sensitivity obtained with DANTE is about a factor 4 , i.e., about a 16-fold gain in experimental time. Note that, the length of the conventional echo sequences is constant in a-e (i.e., $2 \tau_{\text {rot }}$ ), whereas the lengths of the DANTE sequences increase from $26,28,34,34$ to 


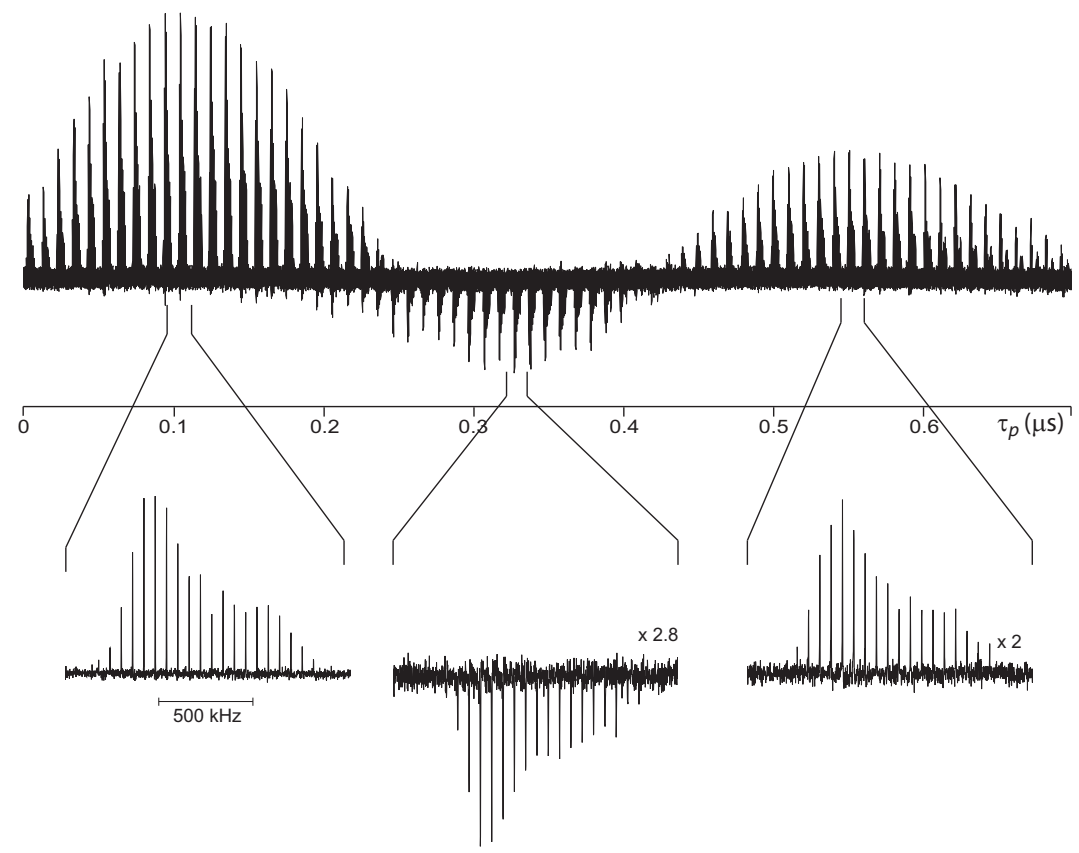

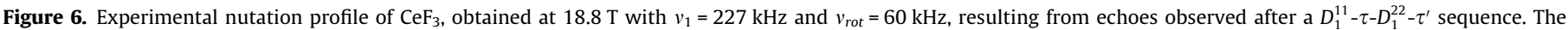

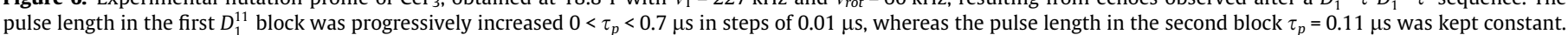

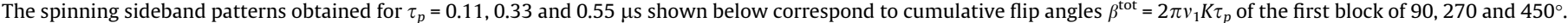
All other experimental parameters were as in Figure 1.

$36 \tau_{\text {rot }}$ in $\mathrm{f}-\mathrm{j}$. This proves that homogeneous decay does not significantly affect the outcome of DANTE schemes in this sample.

\section{Conclusions}

We have shown that DANTE combs of rotor-synchronized pulses can be used to excite and refocus effects of inhomogeneous broadening that extend well over $1 \mathrm{MHz}$. Frequency-dependent phase errors that build up during the dead time before signal acquisition and spurious background signals can be eliminated by forming echoes. The breadth of the global excitation resulting from very short individual pulses with durations $\tau_{p}<1 \mu$ s is maintained for $r f$-field amplitudes as low as $v_{1}=30 \mathrm{kHz}$. The overall duration $K \tau_{\text {rot }}$ of the DANTE combs results in spikelets in the excitation profile that are very selective, thus allowing the excitation of selected sites in samples, for example when signals of different crystallographic environments partially overlap. Arbitrary cumulative flip angles can be obtained with DANTE schemes. This suggests that virtually all rectangular pulses that are commonly used for the creation or transfer of arbitrary coherences in solid-state MAS experiments may be replaced by rotor-synchronized combs of pulses.

\section{Experimental details}

All ${ }^{19} \mathrm{~F}$ MAS spectra of $\mathrm{CeF}_{3}$ have been recorded on a Bruker 800 spectrometer (18.8 T, $752.6 \mathrm{MHz}$ for ${ }^{19} \mathrm{~F}$ ) with an Avance-III console, using $1.3 \mathrm{~mm}$ rotors in a double resonance probe, and a spinning frequency of $v_{\text {rot }}=60 \mathrm{kHz}$. The fluorine chemical shifts were referenced to $\mathrm{CaF}_{2}$ at $-108.6 \mathrm{ppm}$ [32]. Typical $r$-field amplitudes were $14<v_{1}<227 \mathrm{kHz}$. A recycling delay of $0.3 \mathrm{~s}$ was used in all cases. For Dante echoes $D_{N}^{K}-\tau-D_{N}^{K}-\tau^{\prime}$ and $D_{N}^{K}-\tau-D_{N}^{2 K}-\tau^{\prime}$, the delays were set to $\tau=\tau^{\prime}=\tau_{\text {rot }}=16.67 \mu \mathrm{s}$ in all cases. However, numerical simulations show that both delays can be omitted when DANTE schemes are used to perform spin-echo experiments. The Exorcycle [36] phase cycle of the refocusing burst was nested with CYCLOPS
[37] to produce an overall 16-step phase cycle [17]. Numerical simulations were performed with SIMPSON [33], using 256 crystal orientations sampled with the REPULSION scheme [38], and 6 or $8 \gamma$-angles were considered.

\section{Acknowledgements}

This letter was supported by the Swiss National Science Foundation (SNSF), the Ecole Polytechnique Fédérale de Lausanne (EPFL), the Swiss Commission for Technology and Innovation (CTI), and by the Fédération de Recherche (FR-3050) de Très Grandes Infrastructures de Recherche de Résonance Magnétique Nucléaire à Très Hauts Champs (TGIR RMN THC) supported by the French CNRS. Julien Trébosc, Olivier Lafon, and Jean-Paul Amoureux are grateful for funding provided by Region Nord/Pas de Calais, the European Union (FEDER), the CNRS, the French Ministry of Scientific Research, USTL, ENSCL, CortecNet and Bruker BIOSPIN. This letter was supported by Contract ANR-2010-jcjc-0811-01.

\section{References}

[1] E.R. Andrew, A. Bradbury, R.G. Eades, Nature 182 (1958) 1659

[2] E.R. Andrew, A. Bradbury, R.G. Eades, Nature 183 (1959) 1802

[3] I. Lowe, Phys. Rev. Lett. 22 (1959) 133.

[4] M.J. Duer, Solid-State NMR Spectroscopy: Principles and Applications, Blackwell Science, UK, 2002.

[5] M.M. Maricq, S.J. Waugh, J. Phys. Chem. 70 (1979) 3300.

[6] J. Herzfeld, A.E. Berger, J. Chem. Phys. 73 (1980) 6021

[7] M.R. Mitchell, D. Carnevale, R. Orr, K.R. Whittle, S.E. Ashbrook, J. Phys. Chem. C 116 (2012) 4273

[8] M. Castro et al. J. Phys. Chem. C 144 (2010) 12698.

[9] D. Carnevale, V. del Amo, D. Philp, S.E. Ashbrook, Tetrahedron 66 (2010) 6238.

[10] G. Kervern, Angew. Chem. Int. Ed. 48 (2009) 3082.

[11] G. Maruta, S. Takeda, R. Imachi, T. Ishida, T. Nogami, K. Yamaguchi, J. Am. Chem. Soc. 121 (1999) 424.

[12] D. Carlier, M. Ménétrier, C.P. Grey, C. Delmas, G. Ceder, Phys. Rev. B 67 (2003) 174103.

[13] J. Kim, D.S. Middlemiss, N.A. Chernova, B.Y.X. Zhu, C. Masquelier, C.P. Grey, J. Am. Chem. Soc. 132 (2010) 16825.

[14] A. Nayeem, J.P. Yesinowski, J. Chem. Phys. 89 (1988) 4600. 
[15] I. Bertini, C. Luchinat, G. Parigi, Prog. Nucl. Magn. Reson. Spectrosc. 40 (2002) 249.

[16] A.J. Vega, in: D.M. Grant, R.K. Harris (Eds.), Encyclopedia of Nuclear Magnetic Resonance, vol. 4, Wiley, Chichester, 1996.

[17] S. Antonijevic, S. Wimperis, J. Magn. Reson. 164 (2003) 343.

[18] E.L. Hahn, Phys. Rev. 80 (1950) 580.

[19] H.Y. Carr, E.M. Purcell, Phys. Rev. 94 (1954) 630.

[20] G. Bodenhausen, R. Freeman, G.A. Morris, J. Magn. Reson. 23 (1976) 171.

[21] P. Caravatti, G. Bodenhausen, R.R. Ernst, J. Magn. Reson. 55 (1983) 88

[22] V. Vitzthum, M.A. Caporini, S. Ulzega, G. Bodenhausen, J. Magn. Reson. 212 (2011) 234.

[23] V. Vitzthum, M.A. Caporini, S. Ulzega, Julien Trébosc, Olivier Lafon, Jean Paul Amoureux, G. Bodenhausen, J. Magn. Reson. 223 (2012) 228.

[24] S. Antonijevic, S. Wimperis, J. Chem. Phys. 121 (22) (2004) 1.

[25] G. Kervern, G. Pintacuda, L. Emsley, Chem. Phys. Lett. 435 (2007) 157.
[26] E. Kupce, R. Freeman, J. Magn. Reson. 115 (1995) 273.

[27] A.W. MacGregor, L.A. O’Dell, R.W. Schurko, J. Magn. Reson. 208 (2011) 103.

[28] L.A. O’Dell, R.W. Schurko, Chem. Phys. Lett. 464 (2008) 97.

[29] A.J. Pell, G. Kervern, L. Emsley, M. Deschamps, D. Massiot, P.J. Grandinetti, G. Pintacuda, J. Chem. Phys. 134 (2011) 024117.

[30] I. Hung, L. Zhou, F. Pourpoint, C.P. Grey, Z. Gan, J. Am. Chem. Soc. 134 (2012) 1898.

31] C. Bessada, A. Rakhmatullin, A. Rollet, D. Zanghi, J. Nucl. Mater. 360 (2007) 43

[32] R.E. Youngman, C.M. Smith, Phys. Rev. B 78 (2008) 014112.

[33] M. Bak, J.T. Rasmussen, N.C. Nielsen, J. Magn. Reson. 147 (2000) 296.

[34] Z. Gan, J. Am. Chem. Soc. 122 (2000) 3242.

[35] H.-T. Kwak, Z. Gan, J. Magn. Reson. 164 (2003) 369.

[36] G. Bodenhausen, R. Freeman, D.L. Turner, J. Magn. Reson. 27 (1977) 511.

[37] D.I. Hoult, R.E. Richards, Proc. R. Soc. (Lond.) A344 (1975) 311.

[38] M. Bak, N.C. Nielsen, J. Magn. Reson. 125 (1997) 132 